University of Windsor Scholarship at UWindsor

Mechanical, Automotive \& Materials Engineering

Department of Mechanical, Automotive \&

Publications

Materials Engineering

6-10-2019

\title{
Performance evaluation of the remanufacturing system prone to random failure and repair
}

Ronak Savaliya

University of Windsor

Walid Abdul-Kader

University of Windsor

Follow this and additional works at: https://scholar.uwindsor.ca/mechanicalengpub

Part of the Engineering Commons

\section{Recommended Citation}

Savaliya, Ronak and Abdul-Kader, Walid. (2019). Performance evaluation of the remanufacturing system prone to random failure and repair. International Journal of Sustainable Engineering, 1-12.

https://scholar.uwindsor.ca/mechanicalengpub/19 UWindsor. It has been accepted for inclusion in Mechanical, Automotive \& Materials Engineering Publications by an authorized administrator of Scholarship at UWindsor. For more information, please contact scholarship@uwindsor.ca. 


\title{
PERFORMANCE EVALUATION OF REMANUFACTURING SYSTEM PRONE TO RANDOM FAILURE AND REPAIR
}

\begin{abstract}
:
Implementation of new environmental legislation and public awareness has increased the responsibility on manufacturers. Ever since the introduction of remanufacturing, it has been applied in many industries and sectors. However, only $10 \%$ to $20 \%$ of the returned products pass through the remanufacturing process, and the remaining products are disposed in the landfills. Uncertainties like high failure rates of the servers, buffer capacities, and inappropriate preventive maintenance policies would be responsible for most of the delays in remanufacturing operations. In this paper, a simulation based experimental methodology is used to determine the optimal preventive maintenance frequency and buffer allocation in a remanufacturing line, which will help to reduce the cycle time and increase the profit of the firm. Moreover, an estimated relationship between preventive maintenance frequency and Mean Time Between Failure, (MTBF), is presented to determine the best preventive maintenance frequency. The solution approach is applied to computer remanufacturing industry. Analysis of variance, (ANOVA), and regression analysis are performed to denote the influential to obtain the best possible remanufacturing cycle time (performance measures). A case study is used to show the applicability of the modeling approach in assessing and improving the cycle time and the profit of remanufacturing desktop and laptop computers. Managerial insights are highlighted to support managers and decision-makers in their quest for more efficient and smooth operation of the remanufacturing system.
\end{abstract}

\section{Keywords:}

Remanufacturing, Cycle Time, Material Recovery, Preventive Maintenance, Buffer Contribution

\section{Introduction:}

The implementation of rigid environmental legislation and increased public awareness has forced the manufacturers to begin the recycling and remanufacturing of their used products. Remanufacturing is an industrial process that starts with the collection of used products (cores) and restores them to new conditions, and this process allows manufacturers to achieve the same high quality standards of these final products. Furthermore, it reduces the consumption of untapped resources by reusing old materials. Generally, the company pays collection costs to reclaim the 
merchandise (cores) and then, cores will pass through the remanufacturing system such as disassembly, cleaning, inspection, repairing, reassembling, and packing.

Because electronic products have short lifecycles, returned products are time sensitive and any delay in remanufacturing contributes to decreasing the value of the product. For these kinds of products, delay can be expensive as customers are less likely to buy the products that have a short life. In fact, research shows that the returned products generally lose $30 \%$ of their value in process delay, (Guide et al., 2006). For example, time sensitive electronic products like personal computers, (PCs), may lose $1 \%$ of their value per week and the rate is increased at the end of the life cycle. Figure 1 shows the recovered value from returned goods by various remanufacturing processes. It can be seen that if the value of the returned items is $\$ 1000$, then the buyer can only recover $\$ 550$ by various reverse logistic processes and this means that $\$ 450$ is lost due to delay. After a product is sorted into distinct categories like good, (reusable), moderate, (remanufacture), and bad, (recycle), it is crucial to perform remanufacturing operations as fast as possible. Blackburn et al., (2004), found that only $20 \%$ of the returned commodities manage to get higher value and that the remaining $80 \%$ generally drops down to the lower values.

\section{Insert Figure 1 about here}

There are two types of uncertainties related to the delay in process: external and internal. External uncertainties take place due to variations in the quality and quantity of returned materials, changes in demand, and variations in time of product returns. Internal uncertainties incorporate accidental failures of the workstation, inappropriate buffer allocations, high congestion at the facility, and the yield rate of the entire process. The first reason for the delay in processing is the buffer allocation. Buffer capacity controls the work in process for the remanufacturing system. Proper space for work-in-process reduces the probability of "starvation" and "blocking" in the remanufacturing process. In starvation, the buffer is empty and the downstream machine has no parts to process, and therefore, work must stop. Blocking is the condition in which the downstream buffer is filled to its maximum capacity and therefore, this stops the upstream machine in discharging the finished part. Both conditions force the machines to stay idle, which creates an unnecessary delay in the process.

Another reason for work interruption is a sudden failure of the machinery. Any failure needs to be fixed by means of corrective maintenance. Corrective maintenance is a time consuming and costly 
activity, because the maintenance crew needs time to diagnose and repair the failed machine. In fact, increments in buffer capacity beyond the optimal level sometimes decreases the production output more than other factors. In addition, machine availability depends on its MTBF. The MTBF for any machine can be improved by introducing preventive maintenance, (PM). This policy helps to restore the reliability of a machine that has deteriorated over time. Hence, preventive maintenance is necessary to improve the availability and increase the remanufacturing rate.

To summarize, this paper will study the effect of different buffer allocations and different PM frequencies on the cycle time and profit of the remanufacturing line, which is used to remanufacture multiple products. This study will assess machines availability and buffer effectiveness. To achieve this, the current study employs a simulation-based experimental methodology to optimize the buffer allocation and PM by reducing the overall experiment size. This study tackles an important topic of research as remanufacturing industries are trying to increase their revenue by remanufacturing more products. The aim of this paper is to establish an effective way to discover the appropriate PM frequency and optimal buffer allocation so that the performance of the remanufacturing line is maximized and work is flowing without interruption or delays.

\section{Literature Review:}

Very few published works have addressed the buffer allocation issue for the remanufacturing line. But there is no consideration about the preventive maintenance and multi-product production environment. Unlike manufacturing system, remanufacturing systems are characterized by uncertain input and output patterns. The input and output patterns have uncertainty in terms of quality and quantity. Whereas, both the input and the output patterns of a manufacturing system have known quality and quantity, (Esmaeilian et al., 2016). The role of buffer is to decouple two consecutive workstations by providing some freedom so that operations can continue in case of accidental failure and repair, as well as unequal processing times. This is true for both manufacturing and remanufacturing systems. The role of buffers may reduce/ eliminate the phenomena of workstation's blocking and starvation. Blocking phenomenon occurs when the downstream buffer is full and the upstream workstation has no place to unload its processed parts. Starvation phenomenon occurs when a downstream workstation has no parts to work on because the buffer is exhausted and the upstream workstation is down due to failure/ repair. Moreover, to 
Many industries process multiple products. However, due to the complexity of the problem, it has only been explored in scant published works. The issue was firstly analyzed by Abdul-Kader \& Gharbi, (2002). Until this time, it was unacknowledged. In their study, they develop a simulation based experimental design. The methodology was proposed to improve the performance of the manufacturing line. A nonlinear mathematical programming model was employed by AbdulKader et al., (2011) to optimize the buffer capacity for a multi-product production line. They use a lexicographic goal programming method to optimize the buffer capacity. In this model, the authors did not consider explicitly the accidental failure and repair of the machines of the production line. Basically, their model is based on an ideal situation. Jarrahi \& Abdul-Kader, (2015), develop an analytical method to measure the performance of a multi-product unreliable production line with finite buffers between workstations. A decomposition method is then employed to approximate the production rate of the production line. A GI/G/1/N queuing model is applied to obtain parameters such as blocking and starvation probabilities that are needed for the approximation procedure. Like the earlier models, the models cited in this paragraph do not address preventive maintenance. 
The reality is that cycle times are highly dependent on the buffer allocation and preventive maintenance strategy. Other literature on the production system highly concentrate on the optimal buffer inventory during the maintenance duration. Radhoui et al., (2010), find the optimal buffer inventory and optimal PM frequency by using experimental design. Zequeira et al., (2004), define the method to find the optimal time for continuous production period and how to decide the optimal buffer inventory to satisfy demand during downtime. Zequeira et al., (2008), with the goal of cost minimization, use a heuristic method to find the optimal buffer size and PM frequency. Van der Duyn Schouten \& Vanneste, (1995), optimize the PM frequency for two-machines and one buffer production line. They find the optimal way to apply the PM to improve production is by the age of the machine and its wear and tear. None of these models consider a multi-product production environment.

Similarly, Meller and Kim, (1996), also calculate the effect of PM on a two-machine one buffer line. The authors address the impact of preventive maintenance on system cost and buffer size. They study a production system composed of two stations separated by an intermediate buffer where the second station is assumed reliable. The authors assume that failure rate and repair rate of the first machine follow an exponential distribution. In their model and based on certain scenarios, it has been shown that the implementation of a preventive maintenance program decreases the system cost as well as the process variation in terms of output. Noseworthy and Abdul-Kader, (2004), use a simulation based methodology to determine the effect of periodic PM on production costs and overall production rates. Zandieh et al., (2017), propose a genetic algorithm to determine the optimal buffer allocation and period of PM. They use the normal distribution of time to represent the PM in a production line. The goal of their paper is to increase the production rate and minimize the defective rate of products and hereby minimize losses. In contrast, Nahas, (2017), determines the optimal buffer allocation and rate of PM by using a decomposition method. The focus of the above-reviewed papers is on a single-product production environment.

Considering remanufacturing systems, Aksoy \& Gupta, (2005), present a study to optimize buffer allocation for remanufacturing line using a heuristic algorithm. Aksoy \& Gupta, (2011), explore the buffer optimization problem, when servers are on break. They use heuristic algorithm to optimize the buffer allocation. Aksoy \& Gupta, (2010), Su \& Xu, (2014), and Gu, (2018), explore 
buffer allocation problems when servers follow N-policy. They use decomposition principle and expansion methodologies to achieve the goals of remanufacturing and hybrid production lines respectively. Here, N-policy, means that if the quantity of returned products is less than $\mathrm{N}$, then the processor or server either sits idle or continues to work on secondary work. All the literature presented for remanufacturing lines uses the reusable rate of returned product. Moreover, their models do not consider multi-product, set up times, and yield rate, which can be advantageously tackled in simulation modelling techniques given the stochastic nature of the remanufacturing operations and environment.

From the above-indicated limitations of the reviewed publications, this research paper will focus on finding the optimal buffer allocation and PM frequency with the aim to reduce cycle time and increase profit through reusing, remanufacturing, and recovering materials, which are key performance indicators in remanufacturing systems, (Graham et al., 2015). Additionally, consideration of preventive maintenance and multi-product environment is integrated in this comprehensive modelling approach.

The remaining of the paper is organized as follows: Section 3 illustrates working assumptions while Section 4 describes the solution methodology. To demonstrate the applicability and usefulness of the solution approach, a case study is presented in Section 5. Finally, the conclusion and recommendations for future investigations are reported.

\section{Simulation Model Parameters, Assumptions and Performance Measures:}

This section outlines the parameters, working assumptions and performance measures used in the simulation model and experimental design.

\subsection{Process parameters:}

The following symbols are used in this paper. The machines of the remanufacturing system are represented by $M_{i}$, where $i=1,2 \ldots n$. The intermediate buffers are represented by $B_{i}$, where $i=1$, $2 \ldots \mathrm{n}-1$. Products are represented by $\mathrm{P}_{\mathrm{j}}$, where $\mathrm{j}=1,2 \ldots \mathrm{m}$. During the case study, the basic parameters, which are used continuously, are as follow:

$1 / \lambda_{\mathrm{i}}=$ Mean Time Between Failure, $\left(\mathrm{MTBF}_{\mathrm{i}}\right)$, for machine $\mathrm{M}_{\mathrm{i}}$ 


$$
\begin{aligned}
& 1 / \mu_{\mathrm{i}}=\text { Mean Time to Repair, }\left(\mathrm{MTTR}_{\mathrm{i}}\right), \text { machine } \mathrm{M}_{\mathrm{i}} \\
& \mathrm{Z}_{\mathrm{i}}=\text { maximum buffer capacity of buffer } \mathrm{B}_{\mathrm{i}} \\
& \mathrm{T}_{\mathrm{i}}=\text { Time between PM actions } \\
& \mathrm{T}=\text { clock time } \\
& \mathrm{PM}=\text { preventive maintenance } \\
& \mathrm{CM}=\text { corrective maintenance }
\end{aligned}
$$

\subsection{Preventive maintenance calculation:}

As per the study of Meller and Kim, (1996), the long-run MTBF is a function of the operating time between two preventive maintenance actions and the following three parameters:

1. Current MTBF without the PM action, (Minimum)

2. Maximum MTBF with very frequent PM program, (Maximum)

3. $\beta$ - shape factor for the asymptotic gain in PM

The user can calculate the maximum MTBF by mathematical method and approximation method. Maximum MTBF is equated by taking the availability level of $99 \%$ in equation 1 . The shape factor, $\beta$, is estimated and then the time of PM is estimated by using equation 2 .

$$
\begin{gathered}
\mathrm{C}=\frac{M T B F}{M T B F+M T T R} \\
\mathrm{MTBF}=\text { Min. }+(\text { Max. }- \text { Min. })\left(1-\mathrm{e}^{-\beta / T i}\right)
\end{gathered}
$$

\subsection{Performance measures:}

To generate the results and evaluate the serial production line, the following parameters are used in the simulation model as well as in the results.

$$
\begin{gathered}
\mathrm{PO}=\text { Total production output for the remanufacturing system } \\
\mathrm{CT}=\text { Cycle time to finish production of one lot of each product } \\
\mathrm{BZ}_{\mathrm{i}}=\text { Average contents in buffer } \mathrm{B}_{\mathrm{i}} \\
\mathrm{TCM}_{\mathrm{i}}=\text { Total corrective maintenance time during a simulation period or } \\
\text { Unscheduled down time for machine } \mathrm{M}_{\mathrm{i}} \\
\mathrm{TPM}_{\mathrm{i}}=\text { Total PM time during a simulation period or } \\
\text { Scheduled downtime for machine } \mathrm{M}_{\mathrm{i}} \\
\mathrm{BT}_{\mathrm{i}}=\text { Total time machine } \mathrm{M}_{\mathrm{i}} \text { is blocked }
\end{gathered}
$$




\subsection{Cost parameters:}

Various costs, like holding, corrective maintenance, and PM are calculated. All parameters used for the estimation of costs are as follows:

$\mathrm{C}_{\mathrm{r}}=$ Cost of corrective maintenance or cost of unscheduled repair

$\mathrm{C}_{\mathrm{p}}=$ Cost of PM or cost of scheduled repair

$\mathrm{C}_{\mathrm{B}}=$ Operation cost of buffer

$\mathrm{C}_{\mathrm{w}}=$ Wage of employees to operate the remanufacturing system

$\mathrm{C}_{\mathrm{h}}=$ Inventory holding cost

$\mathrm{C}_{\mathrm{u}}=$ Cost of utility to operate the plant

$\mathrm{C}_{\mathrm{e}}=$ Cost of equipment and building

$\mathrm{C}_{\mathrm{c}}=$ Cost of product collection

$\mathrm{TC}=$ Total cost of the remanufacturing system to operate for the defined period

The charge of the corrective maintenance, $\left(\mathrm{C}_{\mathrm{r})}\right.$, depicts the expense to repair the machine when it fails accidentally. It consists of the various fees such as labour, downtime, and maintenance. The maintenance actions that are applied to reduce the random failure of the machines incur the expenditure represented by preventive maintenance cost, $\left(\mathrm{C}_{\mathrm{p}}\right)$. In addition, $\mathrm{PM}$ action includes finances related to oil changes, cleanings, inspections, labour force, and coolants. The buffer operating cost, $\left(\mathrm{C}_{\mathrm{B}}\right)$, represents the money for storing the inventory in buffer, (Meller \& Kim, 1996). The inventory holding cost, $\left(C_{h}\right)$, is for storing and handling the inventory in storage. The utility cost, $\left(\mathrm{C}_{\mathrm{u}}\right)$, includes electricity and general maintenance for the plant. The cost of equipment and building, $\left(\mathrm{C}_{\mathrm{e}}\right)$, represents the charges associated with insurance, fire protection, taxes, building rental, and machine depreciation costs; see Savaliya, (2017).

Handling and utility cost $=\left(\mathrm{C}_{\mathrm{u}}+\mathrm{C}_{\mathrm{h}}\right) \times$ Number of working days

Fixed cost $=$ Handling and utility cost $+\mathrm{C}_{\mathrm{e}}+(\mathrm{Cw} \times$ Number of workers $\times$ Number of working hours)

$\mathrm{TC}=$ Maintenance cost + buffer cost + fixed cost

Profit $=($ Finished Products $\times$ selling price $)-$ TC 


\subsection{Working Assumptions:}

The following assumptions are considered while simulating the model of a remanufacturing system:

1. There is a continuous supply of cores to the first machine, consequently the first machine never gets starved. Accordingly, the last machine of the remanufacturing system has an infinite capacity buffer and so all remanufactured parts can exit the system easily. Hence, the last machine never gets blocked.

2. Two types of cores are remanufactured according to their lot size; (the lot refers to the basic quantity). One type of the cores starts processing when the defined lot of the prior core or product finishes its production. After processing one lot of a product, a machine must be stopped to make necessary adjustments before processing another product. The processing sequence of all the products on any machine is always the same, i.e., product 1 , product $2 \ldots$, product $\mathrm{j}$. For more clarity, the terms cores and products are used interchangeably in this paper.

3. All the machines of the remanufacturing system can process only one work item at a time.

4. All the transitional buffers have finite capacity. The maximum available capacity of a buffer is $\mathrm{Z}_{\mathrm{i}}$.

5. All the machines are prone to unplanned breakdown. The failure rate is defined by $\lambda_{\mathrm{i}}$. The random failure of a machine is usage or operation dependent. This means that if the machine is idle, then it cannot break down.

6. The transferring time of items from one machine to a buffer or from a buffer to a machine is negligible. The loading and unloading of an item onto a machine is small.

7. All the buffers are reliable. They are not subjected to random failures and repairs.

8. Corrective maintenance and PM workers are always available. Corrective maintenance is performed based on first down - first repair rule for any machine.

9. The PM frequency is predefined in the program. A machine undergoes a PM action for the expressed time scheduled.

\section{Methodology:}


Figure 2 illustrates the flow of parts in multi-stage remanufacturing system. The flow chart illustration follows the notation of Section 3 and shows the logic of the simulation model. In multistage remanufacturing lines, each part arrives in the storage. The storage has infinite capacity, and in the multiproduct production environment, all the products arrive as scheduled. As the simulation starts, first product $\mathrm{j}$ will move to machine 1 and at the same time, all the machines will go under the set-up downtime. When the set-up is done, a part will move to machine $\mathrm{M}_{1}$ if the machine is available to accept the part.

The availability of a machine depends on whether it is processing another part, or has failed, or is under preventive maintenance. If the machine is in failed condition, then the machine will be repaired by corrective maintenance and machine downtime will be noted and logged. Ideally in good circumstances, PM is performed as per the schedule. When it is time to perform PM, there is a pause for the maintenance action to be completed, and then the machine will start processing the part. If, however, a machine fails during the processing of a part, it will be repaired and then resume the processing of the unfinished part. Normally, PM is scheduled during the simulation run.

To simplify, a part will be processed on machine $\mathrm{M}_{\mathrm{i}}$ as soon as the machine becomes available. Once the part completes processing on machine $\mathrm{M}_{\mathrm{i}}$, the part is transferred to the downstream buffer if it is not full. If the buffer is full, the part will wait on the machine until a space in the buffer becomes available. At the same time, the blocking time for the machine is logged. A part will be moved out of the buffer according to the first-in-first-out, (FIFO), rule and will progress to the next machine. Once a part finishes processing through all the machines, it will leave the remanufacturing line and will be placed in the inventory. If it is the last part of item $\mathrm{j}$ then the simulation program will determine whether each of the components in the product mix are processed. If all the products are not processed, then it will send the next product batch to the machine and the machine will undergo the setup to process the next product, $\mathrm{j}+1$.

The set-up downtime is used to do the initial preparation for processing the new product. If it is the last part of the product mix, then the cycle time is noted for processing the batches of the product mix. When the run-time of the simulation model is reached, statistics about output results are collected and reported.

\subsection{Solution approach:}


In a multi-product remanufacturing line, there are several factors, (independent variables), which can affect the cycle time and the overall cost of the system. As the number of factors increases, the number of experiments increases as well. Thus, for longer remanufacturing lines, more experiments are needed to assess the performance of the remanufacturing system. Therefore, a mixed level fractional factorial design is considered to estimate the effect of major factors on the performance of the remanufacturing line. The ANOVA will be applied to identify the best contributing factors to the optimal performance of the remanufacturing line. The best results will be optimized further by using the Sim Runner optimizer, which is available in the software package PROMODEL, (2016). The solution approach is shown in Figure 3.

\section{Insert Figures 2 and 3 about here}

By using this solution approach, any remanufacturing line can be optimized. To optimize the remanufacturing line, an experimental design is generated and it will be taken as an input in the simulation model. The simulation model will work as shown in Figure 2 and the results will be analyzed. By statistical analysis of the results, most contributing factors will be decided. The optimal scenario from the experimental design will be identified and taken as an input in Sim Runner for further optimization. Sim Runner will optimize the remanufacturing line parameters. Sim Runner is a built-in optimization module in PROMODEL package, which helps to optimize the objective function within the given boundary conditions. Sim Runner works on the principle of genetic algorithm and evolutionary algorithm. The primary search process of Sim Runner is based on the evolutionary algorithm. The proposed solution approach is applied in the case study to show the usefulness of this approach.

\section{Case study:}

\subsection{Process parameters and assumptions:}

To demonstrate and validate the methodology developed in this paper, a case study is conducted on a personal computer and laptop remanufacturing line. The plant is used to remanufacture, reuse and recycle the laptops and computers. Afterwards, the same remanufacturing line is used to remanufacture both products. The flow of product in the remanufacturing line is shown in Figure 4. The returned products will pass through the inspection and testing operations. At the inspection station, some basic memory and cache tests will be done and the good-quality products will proceed to the labelling station, and the remaining products will go to the testing station. Bad- 
quality products will undergo material recovery operations after finishing the testing operation. The moderate quality products will go to the remanufacturing stage. In the case study, it is assumed that $10 \%$ of the products are in the good-quality category and will proceed to the labelling stations after inspection. On the other hand, $10 \%$ of the inspected products will be found to be unrepairable after the testing operation and will proceed to the material recovery operations.

\section{Insert Figure 4 about here}

The processing time of the returned products highly depends on its quality and the type of problems that need to be fixed during the remanufacturing process. As a result, processing times will vary. The processing time, or the exponential distribution, (e), is used to simulate the large set of possible values. The processing time for each product on each machine is shown in Table 1. As noted previously, each machine will undergo the set-up as the product changes. The set-up time is given in Table 1. All the processing times are taken from Savaliya (2017). Table 1 shows that the remanufacturing, (repairing), stage has higher processing time. Hence, two remanufacturing machines in parallel are used while all the other machine stages have one machine.

\section{Insert Table 1 about here}

As indicated earlier, the machines are subject to random failures and repair. The failure and repair rates for each machine are shown in Table 2.

\section{Insert Table 2 about here}

The collection, labour, utility \& handling, selling, and equipment costs are taken from Savaliya (2017). The holding, utility, and other fixed costs are given in Table 3. The selling price of remanufactured products is depicted in Table 3. By recycling a computer, different materials can be recovered. Therefore, the computers and laptops with the bad quality are sent for material recovery.

\section{Insert Table 3 about here}

The recovery of the computer materials can be very profitable for the firm. The value and the percentage of the recoverable materials in the computer are illustrated in Table 4.

\section{Insert Table 4 about here}

\subsection{Preventive maintenance calculations:}


The failure and repair rates for each machine are indicated in Table 2. To calculate the PM frequency, Equations 1 and 2 defined in Section 3 are used. First, one needs to decide the maximum MTBF of every machine that can be achieved by the frequent PM. It is assumed that a $99 \%$ availability is achieved with PM. Consequently, the availability value is selected $99 \%$ in Equation 1 to calculate the maximum MTBF. Each machine can achieve the availability of $99 \%$ with the maximum value of the MTBF. In Table 5, simple 15 and 35-minute, (or $\mathrm{T}_{\mathrm{i}}$ ), frequencies are selected to apply the PM. To estimate the value of the shape factor, $\beta$, a graph is plotted between MTBF and various shape factor values by using Equation 2 (see also Meller and Kim, 1996). The estimated value of the shape factor, $\beta$, is shown in Table 5.

\section{Insert Table 5 about here}

The parameters of Table 5 are used to find the achievable MTBF for different PM frequencies. Equation 2 calculates the achievable MTBFs for the chosen frequencies and so the achievable MTBFs are displayed in Table 6. The calculation of the MTBF for the inspection machine is given below and remaining MTBFs are calculated by using similar method, (see Equation 2, Section 3.2):

$$
\begin{aligned}
& \operatorname{MTBF}=\operatorname{Min}(\operatorname{MTBF})+(\operatorname{Max}(\operatorname{MTBF})-\operatorname{Min}(\mathrm{MTBF}))\left(1-\mathrm{e}^{-\beta / T i}\right) \\
& \operatorname{MTBF}=1+(10-1)\left(1-\mathrm{e}^{-7.5 / 15}\right)=4.54 \text { hours }
\end{aligned}
$$

In the real world, it is very hard to apply PM in the same time interval. There is always some variation in the defined frequency. The normal distribution is used in the simulation model to account for the effect of variation in PM interval. In this case study, $\mathrm{N}(15,5)$ is used for 15 -minute frequency, where 15 is the average time to apply PM and 5 is the standard deviation. Table 6 shows the normal distributions used in the simulation model and achievable MTBF with this time frequency.

\section{Insert Table 6 about here}

\subsection{Experimental design and analysis of the results:}

Experimental designs are best used to understand the effect of each factor on the remanufacturing line. Fractional factorial design is used to analyze the impact of each factor on cycle time and profit. The level of each factor for fractional design is shown in Table 7. The lower level for 
preventive PM is $\mathrm{N}(15,5)$ minutes as we can reach the maximum MTBF with this frequency. The $\mathrm{N}(35,5)$ minute frequency is chosen as the higher level for the PM. The medium level will have mixed PM frequency. A machine with the highest failure rate will get maintenance that is more frequent and the remaining machines will get less frequent maintenance. In this case study, the inspection and packing machines have a higher failure rate. To be sure, these two machines will get PM at $\mathrm{N}(15,5)$ minute intervals while the remaining machines will go under preventive maintenance at $\mathrm{N}(35,5)$ minute intervals at the intermediate levels of PM.

\section{Insert Table 7 about here}

By using the data of Table 7, a fractional design is modeled. Because of this, a list of 66 different scenarios is obtained to analyze the remanufacturing system. These 66 scenarios are taken as an input in the PROMODEL simulation software. From the results, the cycle time and costs are the response variables representing the performance of the system. The model is simulated for 3500 hours including a warm-up period of 700 hours and replicated 10 times. The warm-up period is determined by using the Welch's moving average method.

\subsection{Analysis of variance, (ANOVA):}

The results of the simulation model are inputted in the MINITAB 2016 statistical software to perform the ANOVA. At this point, ANOVA and regression analysis, (variables in output), is calculated and the results of ANOVA and regression rates are shown in Tables 8 and 9.

\section{Insert Table 8 about here}

The regression analysis report depicts the $\mathrm{R}^{2}=92.43 \%$, which indicates a good fit of the data. In addition, Table 8 shows that the $\mathrm{F}$ ratio is high and equal to 297.12 and it indicates sufficient variation within sample means. The confidence interval value is $95 \%$ in the analysis of the results and the p-value is less than 0.05 which shows the significance of the test.

Next, Table 9 shows the different coefficients of the regression analysis. The analysis indicates that buffer 3(C), buffer 4 (D) and PM frequency (E) have a positive impact on the cycle time. Buffer 4 (D) has the largest impact on the cycle time with $t$-statistics value of -23.384 , which means that buffer 4 is significant to reduce the cycle time. Buffer 4 is followed by the buffer 3 and PM frequency with $t=-14.721$ and $t=-9.066$, respectively. The negative $t$-value denotes the reduction 
in the cycle time due to those factors. Buffer 1 (A) and buffer 2 (B) negatively affect the cycle time of the system as they have the largest positive value of the $t$-statistics. The value of the pstatistics is much lower than the confidence level of 5\%. Therefore, we can conclude that all the factors are significant in the experiment. From the regression analysis, it is easy to see that buffer 2 and buffer 1 should have the lower capacity. Meanwhile, buffer 3, buffer 4, and the PM frequency should have higher values.

\section{Insert Table 9 about here}

By comparing the results of the experimental design, (Table 9), the best scenario was selected and optimized by using the Sim Runner and shown in Table 10. As a result, Table 10, proves that the further optimization of the experimental design results has lowered the average cycle time. Profits for the firm has also increased with the reduction in the buffer size. Therefore, experimental designs are useful in achieving the best PM strategy and optimal buffer allocation. By following the optimal configuration obtained by using Sim Runner, the remanufacturing line can process 17,640 units of computers and laptops in a year. Out of this number, 1760 units are reused, 1580 units are recycled and remaining 14,300 units are remanufactured. Here, the profit is calculated for the overall production of the remanufacturing line. The costs are based on the number of hours of operation, they do not depend on the quantity of products. Therefore, overall profit is generated from good and moderate quality products.

\section{Insert Table 10 about here}

\subsection{Revenue generated by recycling products:}

Table 10 demonstrates the optimal results by Sim Runner and it generates the maximum production. The recycled quantity is selected from the best scenario of Sim Runner to calculate the revenue. Once units are recycled, valuable materials such as gold, silver, and platinum can be recovered. The metal present in a regular computer is given in Table 4. By recycling a computer or laptop, factory owners can generate the average revenue of $\$ 34.26$. Table 11, (below), displays the recycled quantity of each product and the total revenue for the firm. This can generate about $\$ 54,000$ of revenue by recycling a total of 1,580 computers/laptops. Accordingly, instead of consumers throwing their electronic products away, they should be encouraged/motivated to recycle the material and this in turn can be very profitable for a company wishing to use those 


\subsection{Factors analysis and optimization:}

The analysis of factors is accomplished by two methods. The first method is to perform the ANOVA on the results of the experimental design and then determine the factors with the greatest impact on the response variable. For instance, Table 9 shows that buffer 2, buffer 4, and PM have the greatest impact on the dependent variable. This data is enough to decide the optimal range of factors, and these factors should be further optimized using Sim Runner. This is an efficient way to reduce the experiment size and optimize the results.

\section{Insert Figure 5 about here}

On the other hand, the second method of considering data is to examine the interaction plot between the factors and then analyze the effect of each one. For example, Figure 5 shows the interaction plot between the factors and the cycle time. In other words, the interaction plot shows that if buffer 1 and buffer 2 have higher capacities then the remanufacturing line will have the maximum cycle time, (less production). This graph clearly depicts that a PM strategy has the lowest cycle time when the duration of PM is short. The graph illustrates that buffer 4 has the bigger impact over buffer 3 . It also shows that if buffer 4 has the higher value, then we can achieve the lower cycle time by keeping buffer 3 at the lower level. It follows then that buffer 1 and buffer 2 increase the cycle time with higher capacity. Therefore, it is necessary to keep buffer 1 and buffer 2 at lower levels. The interaction of PM frequency and buffer 3 shows that if buffer 3 has a smaller capacity then it minimizes the cycle time. The factor Duration of PM always increases the cycle time. Thus, it is necessary to accomplish the PM action in small durations. After analyzing the interaction plot of the second method, it can be concluded that buffer 1, buffer 2, and buffer 3, should be held at lower levels to achieve the minimum cycle time.

\subsection{Preventive maintenance analysis:}

\section{Insert Figure 6 about here}


There is no mistaking that PM is a very critical factor in the remanufacturing line to reduce accidental failure. It is worth emphasizing again that different PM frequencies are useful to achieve the maximum MTBF. The choice of the best PM frequency is essential to reduce the accidental failure of the machines and subsequent delays without affecting or reducing production output (PO). Moreover, the duration of the PM also affects the cycle time. These points must be clearly established so that the relationship between different PM frequencies and PM durations is understood. Figure 6 represents the relationship between different frequencies of PM and the duration of PM when all the buffers are kept at optimal values. From the graph below, PM frequency shows significant variation in the cycle time with optimal values of the buffer. Figure 6 clearly depicts that as the duration of PM increases, then only 35-minute frequency is necessary to achieve the optimal cycle time. Other frequencies increase the cycle time with increments in duration of PM. Nevertheless, the best results are noticed with even moderate levels of PM. Hence, PM frequency does not only depend on the duration of the PM, but it also depends on the duration of the corrective maintenance.

To continue, Figure 7 depicts the relationship between total maintenance time and repair time. It shows the best frequency to apply PM is when there are different durations of PM and CM. Similarly to PM, when machine downtime decreases in CM, it also improves the output of the system. Here $\mathrm{x}$-axis denotes the repair time. Moving in the right-side direction on the $\mathrm{x}$-axis, the value shows the increment in repair time. If the repair time is doubled, then it is represented by 2. If, however, the repair time is 3 times more than the original repair time then it is represented by 3 on the $\mathrm{X}$-axis. To go on, $\mathrm{Y}$ axis denotes the total maintenance time for the remanufacturing line. The graph is plotted for three different frequencies, which are mentioned in this case study. After analyzing the graph, it is obvious that if PM and CM durations are small, then the manager can use less frequent or failure-based PM. If both durations are high, then the overseer can use a moderate level frequency as mentioned in this case study because it has the least total maintenance time. If CM duration is high and PM duration is small, then a simple 15-minute frequency can be used to minimize the total maintenance time. If the PM is high and the CM duration is small, then managers can use less frequent PM to get a higher production output by reducing maintenance time. 
The duration of CM affects the availability of the machine, which can be represented by Equation 1, (see Section 3.2), where $\mathrm{C}$ is the long-term availability of the machine. From Equation 1, it is plain that any increase in MTTR decreases the availability of the machine. The reduction in availability reduces the uptime of the machine and production output. Thus, not only PM duration but CM duration also has a noticeable role in determining optimal PM frequency. If all machines have different failure rates, then it is better to perform PM on individual machines consistently so the remaining machines may continue to work well from the buffer inventory during that period. This will increase the availability of all the machines and improve the production rate. If, on the contrary, all the machines have similar failure rates, then the manager can perform PM on the whole remanufacturing line at the same time to minimize maintenance time. Overall, the failure rate, repair rate, and duration of PM, should be considered from the start to get the ideal frequency of PM.

\section{Insert Figure 7 about here}

\section{Conclusion:}

This paper addresses the problem of unreliable remanufacturing systems with the purpose of improving performance and reducing or eliminating potential delays that may occur due to accidental failure of equipment. This research also wishes to contribute in the implementing of a more viable pricing policy for remanufactured products. Parameters such as PM duration and frequency, the accidental failure and repair of remanufacturing equipment and buffer allocation, have been considered at length. A simulation-based experimental methodology has been presented to optimize the buffer capacity and frequency of PM, with the aim of improving the cycle time of a remanufacturing line. This methodology has been demonstrated by considering a facility that is remanufacturing desktop and laptop computers. The experimental design of the variables has helped to determine the impact of different buffer sizes on the whole cycle time. The results obtained by simulation have been further optimized by using Sim Runner. This solution approach of experimental design and optimization reduced the overall experiment size. This greatly helps managers obtain the best performance measures within the least amount of time. The results as presented using contour and interactions plots are expressive in terms of reducing the cycle time, increasing the profit, sizing the capacity of each buffer, and determining the best PM duration and 
frequency. Regression and ANOVA tables show a very good fit of the results. These contribute to a better decision-making process and provide managerial insights.

For future work, those in leadership can focus on optimizing the number of workers needed for the task. Furthermore, future research can focus on how PM frequency affects the defective rate of machines and tools that do not operate properly as well as the set-up time. There is no doubt that the MTBF of any machine changes with the change in PM frequency and that any change in the MTBF affects the quality of the work piece (Koren et al., 1998) and set up time of the machines. Without question, this MTBF relationship can and should perform a vital role in order to achieve the highest production rates at the lowest costs.

\section{References}

Abdul-Kader, W., Ganjavi, O., \& Baki, F. (2011). A nonlinear model for optimizing the performance of a multi-product production line. International Transactions in Operational Research, 18 (5), 561-577. http://doi.org/10.1111/j.1475-3995.2011.00814.x

Abdul-Kader, W., \& Gharbi, A. (2002). Capacity estimation of a multi-product unreliable production line. International Journal of Production Research, 40 (18), 4815-4834. http://doi.org/10.1080/0020754021000024148

Aksoy, H. K., \& Gupta, S. M. (2005). Buffer allocation plan for a remanufacturing cell. Computers and Industrial Engineering, 48 (3), 657-677. http://doi.org/10.1016/j.cie.2003.03.007

Aksoy, H. K., \& Gupta, S. M. (2011). Optimal management of remanufacturing systems with server vacations. International Journal of Advanced Manufacturing Technology, 54 (9-12), 11991218. http://doi.org/10.1007/s00170-010-3001-z

Aksoy, H. K., \& Gupta, S. M. (2010). Near optimal buffer allocation in remanufacturing systems with N-policy. Computers and Industrial Engineering, 59 (4), 496-508. http://doi.org/10.1016/j.cie.2010.06.004

Blackburn, J. D., Guide, V. D. R., Souza, G. C., \& Van Wassenhove, L. N. (2004). Reverse supply chains for commercial returns. California management review, 46 (2), 6-22.

Buzacott J. A. (1969). Methods of reliability analysis of production systems subject to breakdowns. In: Grouchko D, Editor. Operations research proceedings of NATO conference, Turin, Italy, June 24-July 4. London: Gordon and Breach Science Publishers, 211-232.

Esmaeilian, B., Behdad, S., Wang, B. (2016). The evolution and future of manufacturing: A review. Journal of Manufacturing Systems, 39, 79-100. http://dx.doi.org/10.1016/j.jmsy.2016.03.001

Gershwin, S.B. \& Schor, J.E. (2000) Efficient algorithms for buffer space allocation. Annals of Operations Research, 2000.93 (1-4), 117-144. 
Graham, I., Goodall, P., Peng, Y., Palmer, C., West, A., Conway, P., Mascolo, J.E. \& Dettmer, F.U. (2015). Performance measurement and KPIs for remanufacturing. Journal of Remanufacturing, 5 (10), 1-17. DOI 10.1186/s13243-015-0019-2

Gu, F. (2018). Performance improvement of remanufacturing systems operating under $N$-policy (Master's thesis), University of Windsor, Canada

Guide, V. D. R., Souza, G. C., Van Wassenhove, L. N., \& Blackburn, J. D. (2006). Time Value of Commercial Product Returns. Management Science, 52 (8), 1200-1214. http://doi.org/10.1287/mnsc.1060.0522

Koren, Yoram, Hu, S. J., \& Thomas W. Weber. (1998). Impact of manufacturing system configuration on performance. CIRP Annals-Manufacturing Technology 47.1, 369-372.

Jarrahi, F. \& Abdul-Kader, W. (2015). Performance evaluation of a multi-product production line: An approximation method. Applied Mathematical Modelling, 39 (13), 3619-3636. http://dx.doi.org/10.1016/j.apm.2014.11.059

Meller, R.D \& Kim, D. S. (1996). The impact of preventive maintenance on system cost and buffer size. European Journal of Operational Research, 95, 577-591.

Nahas, N. (2017). Buffer allocation and preventive maintenance optimization in unreliable production lines. Journal of Intelligent Manufacturing, 28 (1), 85-93.

Noseworthy, S., \& Abdul-Kader, W. (2004). Impact of preventive maintenance on serial production line performance: a simulation approach. ASAC 2004.

Radhoui, M., Rezg, N., \& Chelbi, A. (2010). Joint quality control and preventive maintenance strategy for imperfect production processes. Journal of Intelligent Manufacturing, 21 (2), 205212. http://doi.org/10.1007/s10845-008-0198-z

Ravi, V., Shankar, R., \& Tiwari, M. K. (2005). Analyzing alternatives in reverse logistics for endof-life computers: ANP and balanced scorecard approach. Computers \& industrial engineering, 48 (2), 327-356.

Savaliya.R. (2017). Performance evaluation of remanufacturing systems (Master's thesis), University of Windsor, Canada

Su, C., \& Xu, A. (2014). Buffer allocation for hybrid manufacturing/remanufacturing system considering quality grading. International Journal of Production Research, 52 (5), 1269-1284. http://doi.org/10.1080/00207543.2013.828165

Van der Duyn Schouten, F. a., \& Vanneste, S. G. (1995). Maintenance optimization of a production system with buffer capacity. European Journal of Operational Research, 82 (2), 323-338. http://doi.org/10.1016/0377-2217(94)00267-G

Zandieh, M., Joreir-Ahmadi, M. N., \& Fadaei-Rafsanjani, A. (2017). Buffer allocation problem and preventive maintenance planning in non-homogenous unreliable production lines. The 
International Journal of Advanced Manufacturing Technology. http://doi.org/10.1007/s00170016-9744-4

Zequeira, R. I., Prida, B., \& Valdes, J. E. (2004). Optimal buffer inventory and preventive maintenance for an imperfect production process. International Journal of Production Research, 42 (5), 959-974. http://doi.org/10.1080/00207540310001631610

Zequeira, R. I., Valdes, J. E., \& Berenguer, C. (2008). Optimal buffer inventory and opportunistic preventive maintenance under random production capacity availability. International Journal of Production Economics, 111 (2), 686-696. http://doi.org/10.1016/j.ijpe.2007.02.037 


\section{List of Figures}

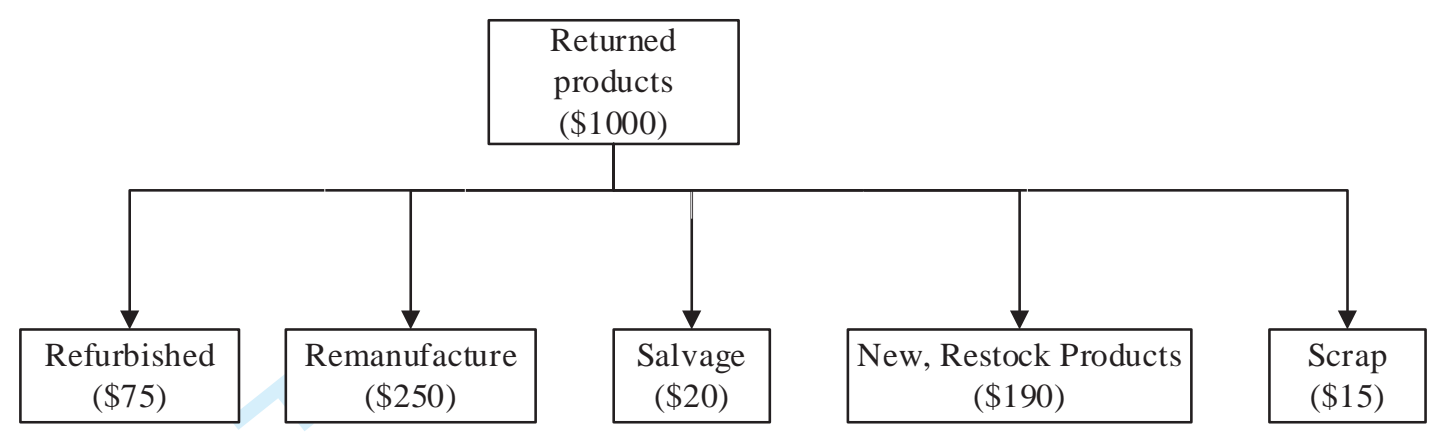

Figure 1: Revenue generated from various remanufacturing processes, adapted from Blackburn et al., 2004 


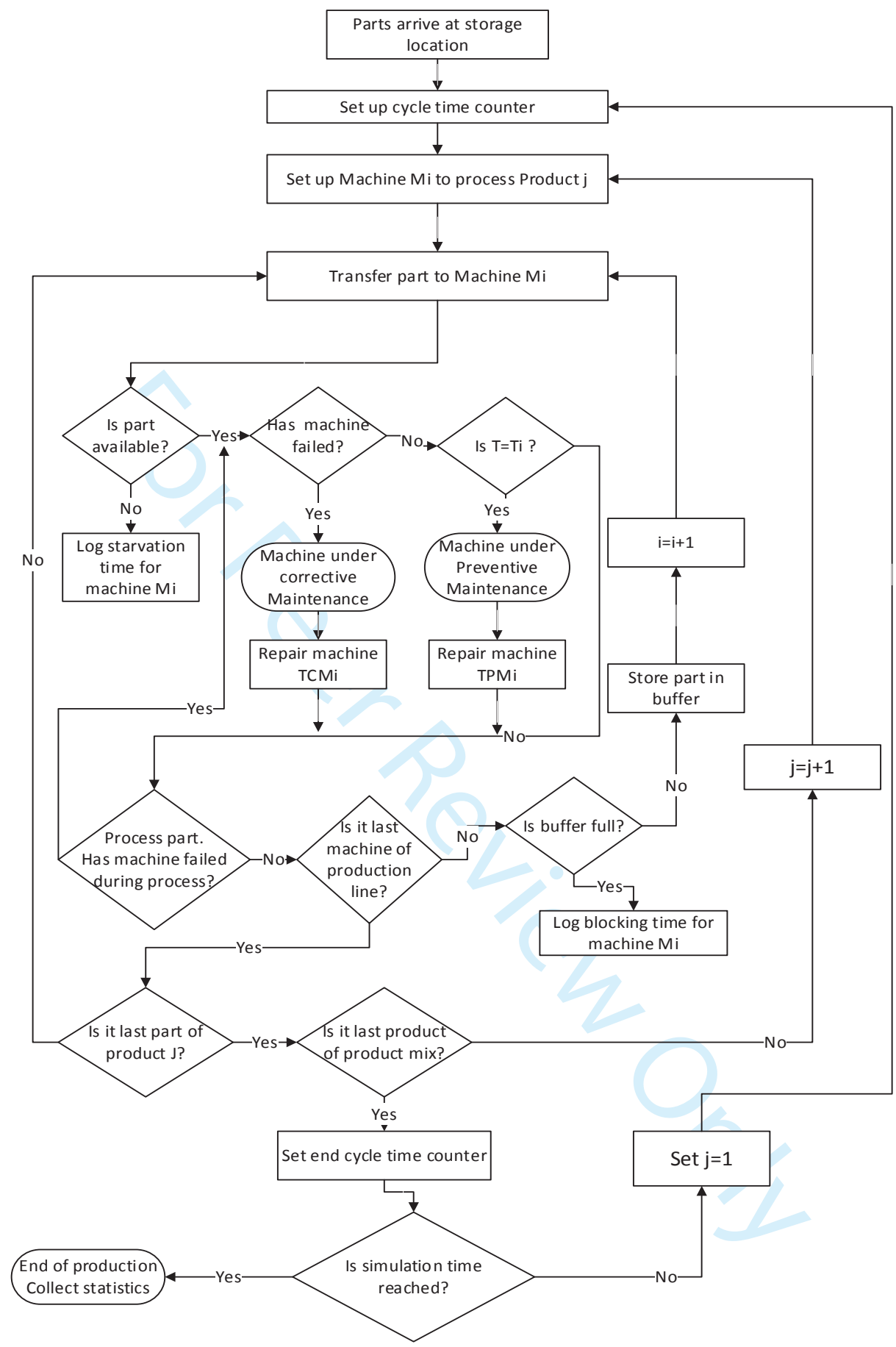

Figure 2: Flow chart of simulation model 


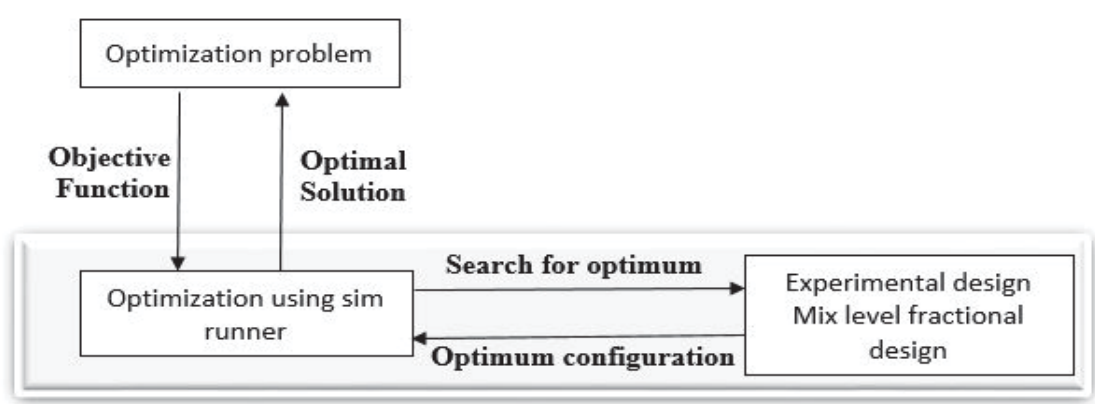

Figure 3: Solution Approach

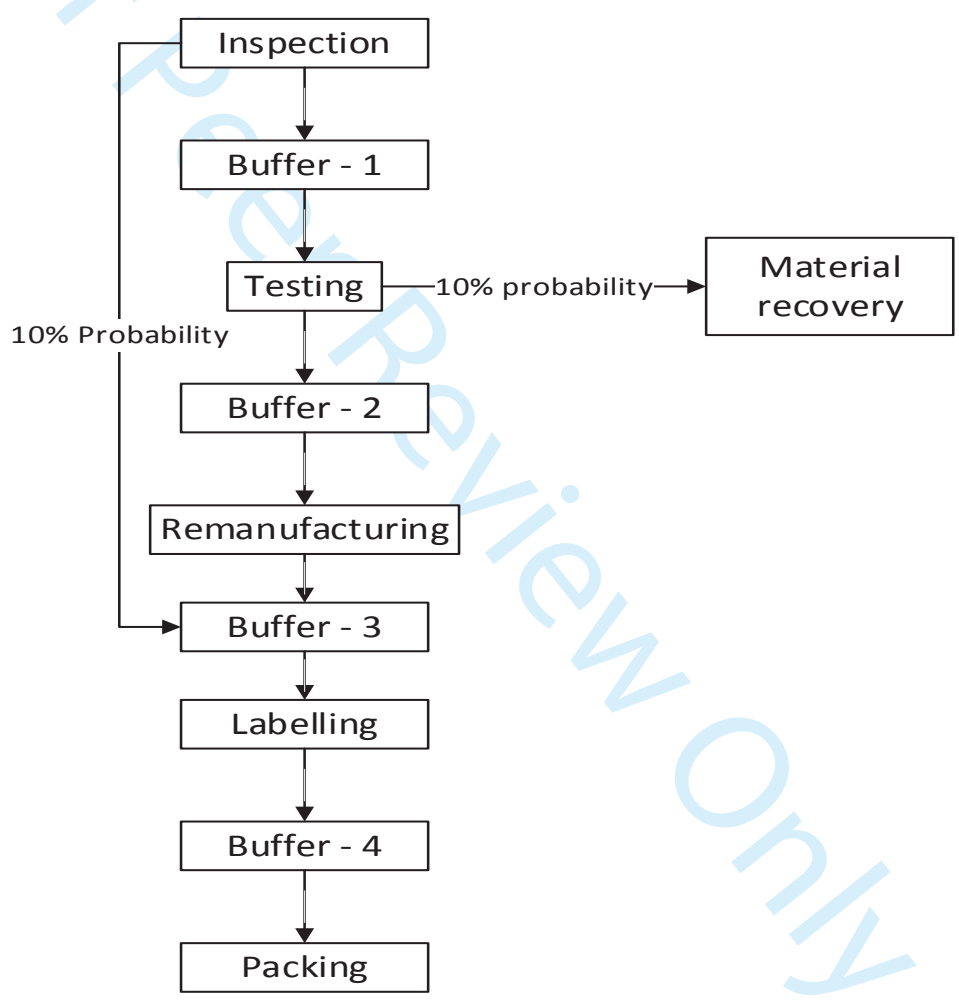

Figure 4: Flow of product in computer and laptop remanufacturing line 


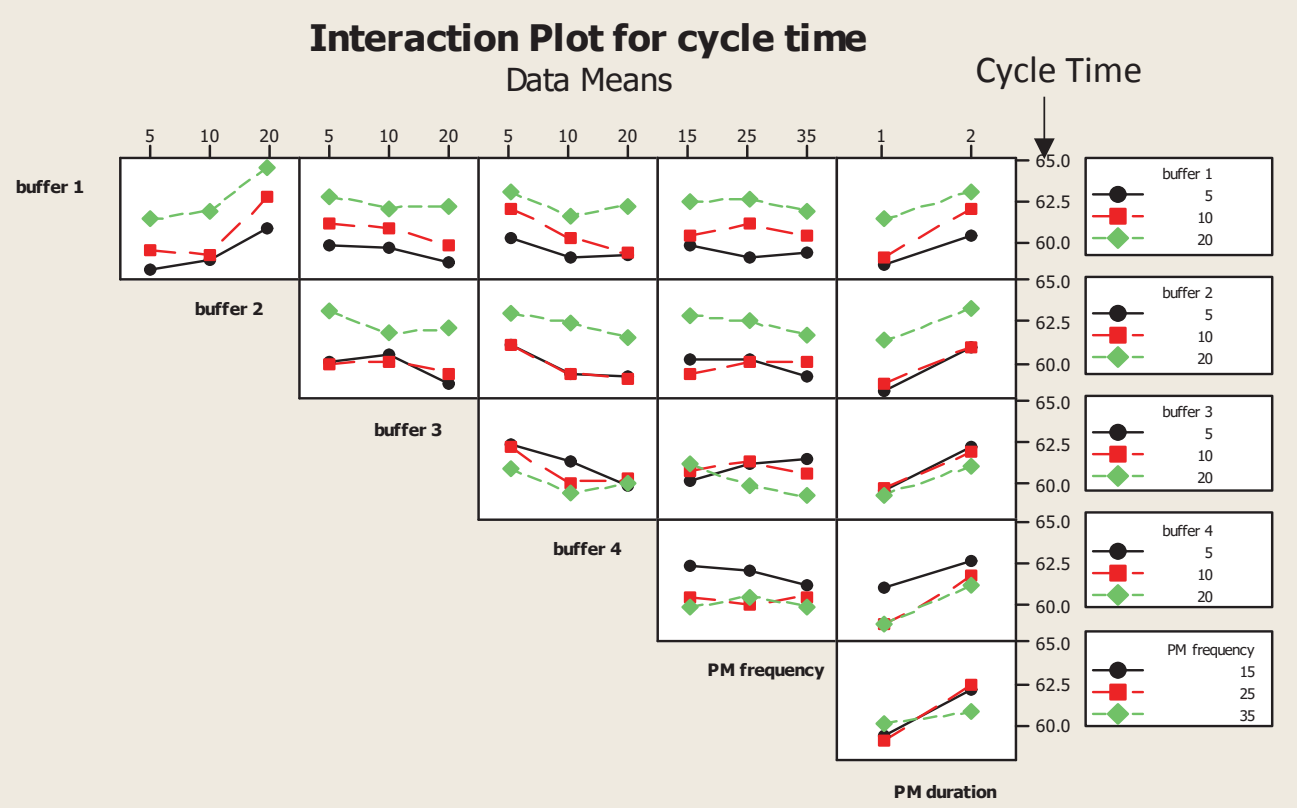

Figure 5: Interaction plot for cycle time

Contour Plot of cycle time vs PM duration, PM frequency
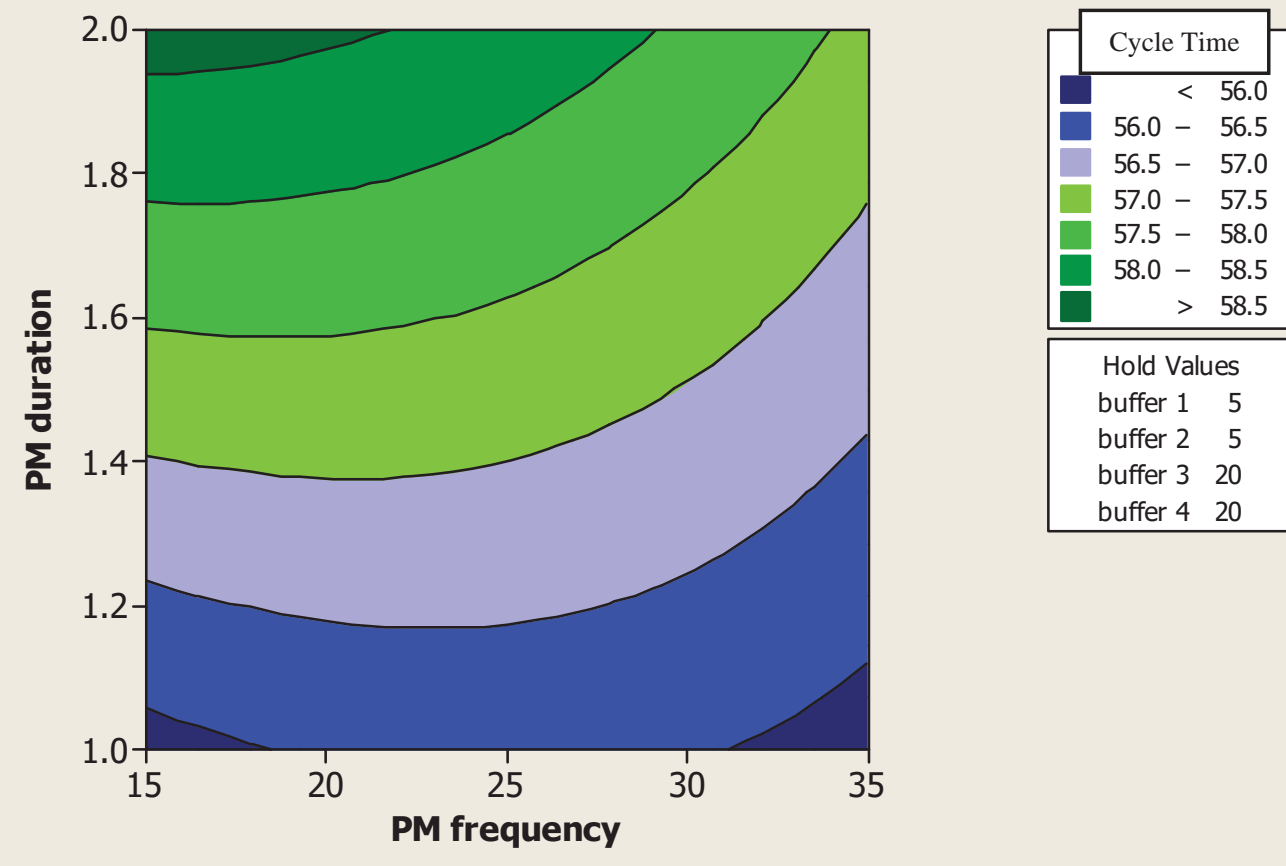

Hold Values buffer 15 buffer 25

buffer 320

buffer $4 \quad 20$

Figure 6: Contour plot of cycle time 
Figure 7: Total maintenance time vs repair time

\section{$\underline{\text { List of Tables }}$}

Table 1: Processing times for computer and laptop remanufacturing line, (taken from Savaliya (2017))

\begin{tabular}{|l|l|l|l|l|l|l|}
\hline & Set up & Inspection & Testing & $\begin{array}{l}\text { Remanu- } \\
\text { facturing }\end{array}$ & Labelling & Packing \\
\hline Computer & 30 & e (1.23) & e (7.32) & e (20) & e (5.66) & e (9.1462) \\
\hline Laptop & 30 & e (1.05) & e (6.5) & e (15) & e (5.66) & e (9.1462) \\
\hline
\end{tabular}

Table 2: Failure and repair rates

\begin{tabular}{|l|l|l|}
\hline Work station & Failure rate, $\boldsymbol{\lambda}$ (hours) & Repair rate, $\boldsymbol{\mu}$ (hours) \\
\hline Inspection & 1 & 10 \\
\hline Testing & 1.33 & 12 \\
\hline Remanufacturing & 0.86 & 7.5 \\
\hline Labelling & 0.75 & 6 \\
\hline Packing & 1 & 8.6 \\
\hline
\end{tabular}




\section{Table 3: Cost associated with computer and laptop}

\begin{tabular}{|l|l|}
\hline Category of cost & Cost \\
\hline Worker & $\$ 15$ per hour \\
\hline Utility and holding cost & $\$ 93$ per day \\
\hline Collection cost of computer & $\$ 150$ per computer \\
\hline Collection cost of laptop & $\$ 200$ per laptop \\
\hline Equipment cost & $\$ 811000$ per year \\
\hline Corrective maintenance cost & $\$ 100$ per hour \\
\hline Preventive maintenance cost & $\$ 10$ per hour \\
\hline Space cost & $\$ 0.2774$ per sq. feet / hour \\
\hline Selling price of computer & $\$ 250$ per computer \\
\hline Selling price of laptop & $\$ 400$ per laptop \\
\hline
\end{tabular}

Table 4: Recoverable materials in computer, taken from Savaliya, 2017

\begin{tabular}{|l|l|l|}
\hline Material & Percent & Value (\$) \\
\hline Plastics & 23.00 & 11.73 \\
\hline Aluminum & 6.30 & 9.11 \\
\hline Steel & 20.50 & 4.18 \\
\hline Gold & 0.001 & 6.27 \\
\hline Silver & 0.02 & 1.03 \\
\hline Lead & 6.30 & 1.93 \\
\hline Cadmium & 0.01 & 0.01 \\
\hline Mercury & 0.0022 & 0.00 \\
\hline Total & $\mathbf{5 6 . 1 3}$ & $\mathbf{\$ 3 4 . 2 6}$ \\
\hline
\end{tabular}

Table 5: Parameters of preventive maintenance

\begin{tabular}{|c|c|c|c|c|c|}
\hline & Inspection & Testing & Remanufacturing & Labelling & Packing \\
\hline MIN. & 1 & 1.33 & 0.86 & 0.75 & 1 \\
\hline MAX. & 10 & 8.33 & 15 & 11.66 & 13.33 \\
\hline$\beta$ & 7.5 & 12.5 & 27 & 27 & 6 \\
\hline
\end{tabular}

Table 6: MTBF and preventive maintenance frequency

\begin{tabular}{|l|l|l|l|l|l|}
\hline Frequency & Inspection & Testing & Remanufacturing & Labelling & Packing \\
\hline $\mathrm{N}(15,5) \mathrm{min}$ & 4.54 & 5.3 & 13 & 10 & 5.9 \\
\hline $\mathrm{N}(35,5) \mathrm{min}$ & 2.7 & 3.4 & 8 & 6.5 & 2.9 \\
\hline
\end{tabular}


Table 7: Factors and their levels for experimental design

\begin{tabular}{|l|c|c|c|}
\hline Factor & Low & Medium & High \\
\hline Buffer 1 (A) & 5 & 10 & 20 \\
\hline Buffer 2 (B) & 5 & 10 & 20 \\
\hline Buffer 3 (C) & 5 & 10 & 20 \\
\hline Buffer 4 (D) & 5 & 10 & 20 \\
\hline PM Freq. (E) & 15 & 25 & 35 \\
\hline PM Duration(F) & 1 & & 2 \\
\hline
\end{tabular}

Table 8: Analysis of variance, (ANOVA)

\begin{tabular}{|c|c|c|c|c|c|}
\hline$S=0.674210$ & & & & & \\
\hline $\mathrm{R}-\mathrm{Sq}=92.43$ & & & & & \\
\hline R-Sq. $(\operatorname{adj})=$ & $92.12 \%$ & & & & \\
\hline Source & DF & SS & MS & $\mathbf{F}$ & P-value \\
\hline Regression & 26 & 3511.47 & 135.057 & 297.12 & 0.000 \\
\hline Residual Error & 633 & 287.74 & 0.455 & & \\
\hline Total & 659 & 3799.21 & & & \\
\hline
\end{tabular}

Table 9: Coefficients of regression analysis

\begin{tabular}{|c|c|c|c|c|}
\hline Parameters & Coefficient & SE coefficient & $\boldsymbol{t}$ & P-value \\
\hline Constant & 60.2621 & 0.11689 & 515.548 & 0.000 \\
\hline A & 1.5988 & 0.03610 & 44.290 & 0.000 \\
\hline B & 1.6803 & 0.03692 & 45.508 & 0.000 \\
\hline C & -0.5732 & 0.03894 & -14.721 & 0.000 \\
\hline D & -0.8095 & 0.03462 & -23.384 & 0.000 \\
\hline E & -0.3299 & 0.03639 & -9.066 & 0.000 \\
\hline F & 1.1336 & 0.02908 & 38.989 & 0.000 \\
\hline
\end{tabular}

Table 10: Optimized results for remanufacturing line

\begin{tabular}{|l|c|c|c|c|c|c|c|c|}
\hline Result type & A & B & C & D & E & F & Cycle time (hours) & Profit (\$) \\
\hline Experimental design & 5 & 5 & 5 & 20 & 25 & 1 & 56.53 & 288,817 \\
\hline Sim Runner & 1 & 4 & 5 & 20 & 25 & 1 & 55.19 & 316,948 \\
\hline
\end{tabular}

Table 11: Revenue generated by recycling computers and laptops

\begin{tabular}{|c|c|c|}
\hline Computer (units) & Laptops (units) & Revenue (\$) \\
\hline 886 & 694 & $\sim \$ 54,130.00$ \\
\hline
\end{tabular}

